\title{
Horses for courses. Comparative gastroenterology: common ground and collaborative potential
}

\author{
Neil P H Hudson, Malcolm G Dunlop
}

Gastrointestinal Motility and Disease Laboratory, Royal (Dick) School of Veterinary Studies, University of Edinburgh,

Summerhall, Edinburgh EH9 $1 \mathrm{QH}$ Neil P H Hudson veterinary fellow

Academic Coloproctology, University of Edinburgh, Western General Hospital Edinburgh EH4 2XU

Malcolm G Dunlop professor

Correspondence to: N Hudson neil.hudson@ed.ac.uk BMJ 2005;331:1248-51
Gastrointestinal diseases, including motility and obstructive disorders, are common in both human ${ }^{1}$ and veterinary ${ }^{2}$ medicine. Such disorders tend to be clinically important problems in both disciplines, with extensive impact on veterinary practice as well as having human public health and economic implications. Considerable time is lost from work due to gut disorders. Motor disorders of the gastrointestinal tract are particularly common, and understanding the physiology of motility and gut function is key to examination of the pathophysiology of intestinal disease. ${ }^{3}$ Few animal models of functional gastrointestinal disorders exist, however, and so there is considerable rationale for comparative study of disease in veterinary and human medicine. Such study affords the opportunity to shed new light on disease processes that so far have proved difficult to study for practical, ethical, and biological reasons. Comparable intestinal disorders between species include postoperative ileus, intestinal obstruction, pseudo-obstruction, irritable bowel syndrome, and inflammatory bowel diseases.

\section{Sources and selection criteria}

We used personal archives of references and, when appropriate, carried out PubMed searches on relevant veterinary and human gastroenterological topics.

\section{Constipation and pseudo-obstruction syndromes}

Pseudo-obstruction and enteric dysmotility syndromes are increasingly being recognised in medical gastroenterology and are thought to represent an impairment of the intrinsic neuromuscular or extrinsic control of gut motility. ${ }^{45}$

Constipation is one of the most common digestive disorders in humans ${ }^{\mathrm{w} 1}$ and, therefore, animal examples such as feline constipation provide opportunity for novel insight into disease mechanism in humans, free from confounding effects accumulated over the lifespan of a human. Feline constipation is common in small animal veterinary practice. Acquired idiopathic megacolon occurs in middle aged to older cats. The pathogenesis is unclear but may involve an impairment of colonic smooth muscle function. ${ }^{6}$ The feline syndrome appears to bear many similarities to megacolon in humans. ${ }^{7}$ In the horse, a case of myenteric ganglionitis classified as an equine form of chronic idiopathic intestinal pseudo-obstruction has been reported. ${ }^{\text {w2 }}$ In dogs, dysmotility syndromes are rarer but include chronic intestinal pseudo-obstruction and functional intestinal hypomotility associated with myenteric neuronal disease. In humans, the majority of pseudo-obstruction that is not associated with electrolyte imbalance or trauma is also neurogenic. ${ }^{4}$ In contrast, gut myopathy is the most common single observation in chronic pseudo-obstruction. ${ }^{8}$ Thus, there are useful paradigms in veterinary practice that

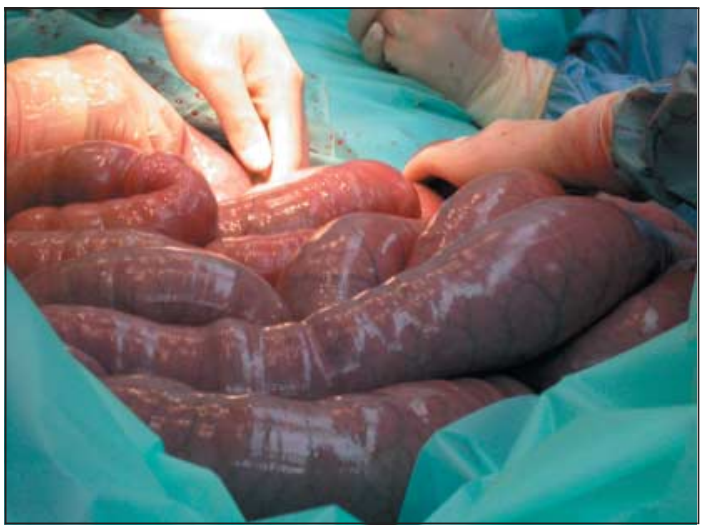

Fig 1 Exploratory laparotomy in a horse with grass sickness showing noticeable small intestinal distension

could lead to novel insight, even through descriptive research that would not be possible in human practice.

\section{Dysautonomia}

Dysautonomias occur in several species including the cat, dog, hare, rabbit, and humans. ${ }^{9}{ }^{\text {w3 }}{ }^{\text {w4 }}$ An important syndrome in veterinary medicine is grass sickness (equine dysautonomia; fig 1), which is a common and often fatal disease of unknown cause characterised by autonomic dysfunction of the alimentary tract of equids kept at grass. ${ }^{10}$ w $^{5}$ Presenting clinical signs include colic (abdominal pain), ileus, anorexia, dysphagia, tachycardia, excessive salivation, patchy sweating, ptosis, and rhinitis sicca. The disease varies in duration and severity of clinical signs, from acute through to chronic manifestations. In certain chronic mild cases, recovery is possible with intensive nursing. ${ }^{w 5-w 7}$ The cause remains enigmatic, but study of the basic mechanisms of grass sickness ${ }^{10}$ holds potential to inform human studies aimed at understanding chronic gut motility syndromes thought to have a post-infective component.

\section{Hirschsprung's disease and lethal white foal syndrome}

Hirschsprung's disease is a human congenital intestinal aganglionosis characterised by signs of intestinal obstruction or severe chronic constipation. ${ }^{911}$ Cardinal features are an absence of ganglion cells and their intrinsic nerve fibres and proliferation of extrinsic nerve fibres. ${ }^{9}$ Mutations in several genes have been causally implicated, including RET, endothelin-3, endothelin-B receptor, Sox10, and SMADIP1. ${ }^{11}$ Ileocolonic aganglionosis (lethal white foal syndrome), involving the absence of myenteric ganglia in the white progeny of overo spotted horses, has been described. This disease could be considered an equine version of the human Hirschs-

References w1 to w23 are on bmj.com 
prung's disease and is associated with a mutation of the endothelin-B receptor gene. ${ }^{\text {w8 }}$ Murray et al have reported a case of a foal with megacolon with myenteric hypoganglionosis that, unlike the lethal white foal syndrome, was not a pigment related disorder. ${ }^{\mathrm{w} 9}$ Thus, study of the genetic basis of such equine disorders may allow active breeding programmes to eradicate disorders that may be due to variable penetrance alleles segregating in animal stocks.

\section{Intestinal obstruction and colic}

Impaired transit through the gastrointestinal tract usually results from mechanical obstruction, but failure of normal motility (ileus) is also important. ${ }^{1}$ In human intestinal obstruction and ileus, patients present with varying degrees of colicky abdominal pain, tenderness and distension, vomiting, and constipation. ${ }^{1}$ In equine medicine, colic (fig 2) is reported by insurance companies and universities as the single greatest killer of horses. ${ }^{12}$ In a multicentre study of 4279 cases of colic, $35 \%$ were classified as obstructions, $21 \%$ as strangulating obstructions, and 25\% as undiagnosed colics. ${ }^{12}$ Colic in the horse ranges from mildly painful cases that may resolve with little or no medical therapy through to severe forms that may require surgery. Although some causes of colic are clearly identifiable at surgery, the cause of many disease processes resulting in colic in horses remains complex and often enigmatic. ${ }^{12}$ w10 Similarly in humans, idiopathic cramping abdominal pain is a frequent accompaniment of the enigmatic irritable bowel syndrome. ${ }^{\mathrm{w} 11}$ Although irritable bowel syndrome has not been documented in the horse, its existence seems plausible, if not likely, when considering the large number of cases of colic and chronic diarrhoea that remain unelucidated. ${ }^{\mathrm{w} 10}$ w12 Irritable bowel syndrome has been described in the dog, with clinical signs that may include diarrhoea, intermittent bloating, nausea, vomiting, and abdominal pain. ${ }^{13}{ }^{\text {w13 }}$ Hence there are considerable parallels for study of the pathogenesis of motility disorders associated with colic or diarrhoea, or both, in both human and animal practice. Clinical examples may be useful in addition to experimental models such as the recently described rectal instillation of butyrate in the rat to study irritable bowel syndrome. ${ }^{14}$

\section{Postoperative ileus}

Ileus is the syndrome of functional inhibition of propulsive bowel motility, ${ }^{\mathrm{w} 14}$ most commonly arising in the immediate postoperative period after intraperitoneal surgery. ${ }^{1}$ In all species, postoperative ileus remains a poorly understood clinical problem, and the lack of a specific therapy means that it has an important economic impact because of prolonged hospital stay. ${ }^{\text {w14 }}$ Postoperative ileus is thought to be the result of loss of normal coordination of intestinal contraction by the intrinsic electrical activity of the gut. ${ }^{1}$ Manipulation of bowel leads to local inflammation and impaired muscle function. ${ }^{\text {w15 }}{ }^{\text {w16 }}$ It has been suggested that postoperative ileus is mediated through a leucocytic inflammatory response. ${ }^{\mathrm{w} 17}$ Jejunal manipulation in the rat causes a pan-enteric inflammation and prolonged dysmotility. ${ }^{15}$ This concept has recently been examined in the horse, where serosal and neuromuscular inflammation was observed in tissues harvested 18 hours after laparotomy and deliberate intestinal manipulation and ischaemia. ${ }^{16}$ Equine postoperative ileus is common and often fatal. In a retrospective study of 259 horses that had undergone abdominal surgery for colic, postoperative ileus accounted for $43 \%$ of the 84 postoperative deaths. ${ }^{17}$ Much progress has been made in reducing the development of postoperative ileus in humans, including the avoidance of starch in gloves, optimised postoperative fluid and electrolyte therapy, and early feeding, as well as attempts at chemical means to reduce adhesion formation. Therefore much can be, and already has been, learnt from the human disorder to inform research in equine surgical practice. Similarly, novel understanding could be gained of postoperative ileus in humans by using the horse as a model for the condition, not least because of access to tissues from frequent deaths in the clinical setting.

\section{Inflammatory bowel diseases}

Inflammatory bowel diseases are chronic inflammatory disorders affecting an increasing number of human patients, the main forms being Crohn's disease and ulcerative colitis. Although the cause remains unclear, research is focusing on genetic, inflammatory, immunological, and infectious factors. ${ }^{\text {w18-w20 }}$ In veterinary medicine inflammatory bowel diseases have been described in a range of species including the dog, cat, and horse, with many similarities to the conditions in humans. ${ }^{18 \text { w21 w22 }}$ As genetic loci contributing to the cause of inflammatory bowel disease are discovered, ${ }^{19}$ it becomes possible to study the homologous genes in animals in order to inform breeding strategies, as well as allowing possibilities of generating "natural" comparable animal models through selective breeding approaches.

\section{Comparative gastroenterological research}

Comparative research offers mutual benefits to researchers working in human and veterinary medicine, thus benefiting both human and animal patients. Research modalities such as immunohistochemistry, tissue culture, electrophysiology, and molecular biology are widely used in both disciplines.

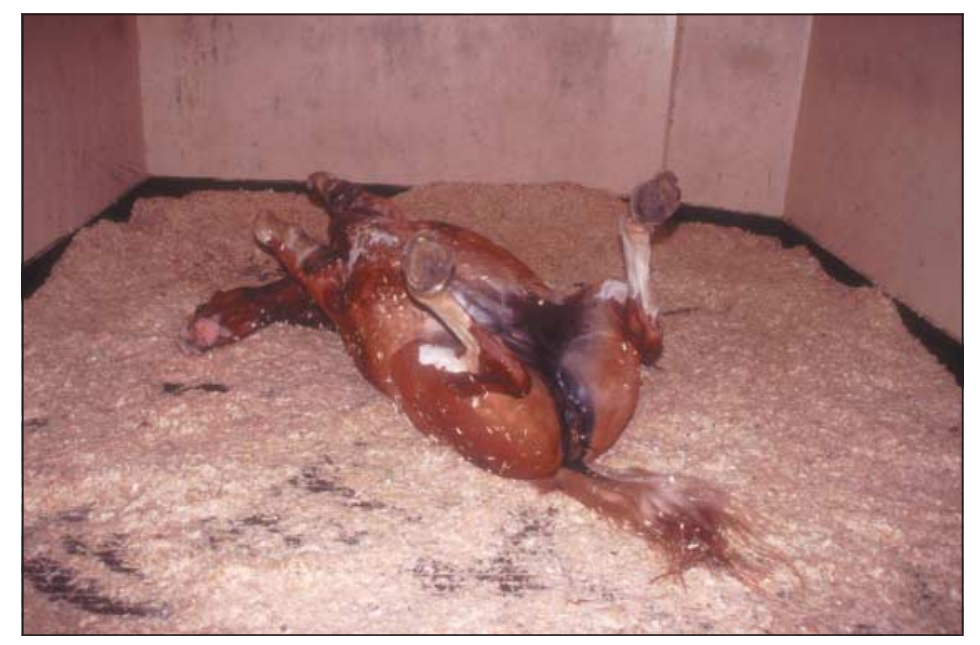

Fig 2 Horse showing signs of severe colic 
Enteric nervous system, interstitial cells of Cajal, and immunohistochemistry

The enteric nervous system has a central role in the control of most gastrointestinal functions. Immunohistochemistry is a useful research tool for investigating the anatomy and function of this system. Much of the knowledge of the immunohistochemistry of the enteric nervous system has been gathered from the guinea pig, but there is an increasing amount of work in other species such as the pig and in humans.

The interstitial cells of Cajal are the pacemakers and mediators of neurotransmission in the gastrointestinal tract. These cells have been implicated, either primarily or secondarily, in the pathogenesis of gastrointestinal disease processes in which there is a prominent element of disturbance to motility.

Antibodies to c-Kit protein are now readily available for the immunohistochemical labelling of the interstitial cells of Cajal. Abnormalities of these cells have been associated with disorders of the human gastrointestinal system, including Hirschsprung's disease, infantile hypertrophic pyloric stenosis, ulcerative colitis, chronic idiopathic intestinal pseudoobstruction, slow transit constipation, and Chagas' disease. ${ }^{20}$ To date, the only studies of the interstitial cells of Cajal in the numerous veterinary gastrointestinal disorders have been in the horse, implicating their involvement in grass sickness ${ }^{21}$ and obstructive colic. ${ }^{22}$

\section{Electrophysiology}

The interstitial cells of Cajal are the initiators of slow waves in the gastrointestinal tract. Slow wave activity is the rate limiting step for peristaltic activity. Slow waves can be recorded in vitro using microelectrodes to record electrical activity in individual smooth muscle cells. Recordings have been made in a range of species such as the cat, dog, and mouse. Limited experiments have been carried out on porcine, human, and equine intestinal smooth muscle. Good correlation exists between findings in vitro and the observed contractile properties in vivo. Pacemaking in human intestine bears many similarities to that in other species such as the pig, cat, and dog. Indeed, the pig is a useful model, not only because of the abundant supply of abattoir derived tissue, but because intestine from the pig possesses functional and pathological similarities to human intestine. ${ }^{23}$ To date there have been few in vitro studies of the electrical activity of diseased intestine. Kubota et $\mathrm{al}^{24}$ found that the aganglionic segments of colon in Hirschsprung's disease were electrically quiescent. Hudson et $\mathrm{al}^{25}$ found that the intestine of horses with grass sickness exhibited prominent slow wave activity despite severe damage to the enteric neurones. Therefore, comparative electrophysiological studies in health and disease represent a novel approach to examining the physiology and pathophysiology of intestinal function.

\section{Tissue culture and molecular biology}

The development of techniques for culturing the enteric nervous system has led to greater understanding of gut physiology. Cultures are useful for correlating the morphological, biophysical, pharmacological, and synaptic properties of neurones, and for testing the ability of altered environmental conditions to change these properties. Therefore, culture systems offer considerable

\section{Summary points}

Across species there are common elements in gastrointestinal diseases that may include postoperative ileus, intestinal obstruction, pseudo-obstruction, irritable bowel syndrome, and inflammatory bowel diseases

Useful paradigms in veterinary practice could lead to novel insights in human practice

Collaboration between the medical and veterinary professions has benefits, including the two way transfer of clinical and research knowledge

Comparative gastroenterological research has considerable potential to benefit both human and animal patients

potential for the development of in vitro models for diseases of the enteric nervous system. Myenteric neurones have been cultured from the human large intestine, but there are few reports of equine enteric neurones being grown in tissue culture. ${ }^{23}$

Molecular biological approaches have allowed closer analysis of gastrointestinal function, including investigation of expression of the c-Kit protein (encoded by the c-kit gene) in the interstitial cells of Cajal. Information about the genetic basis of gastrointestinal diseases is expanding rapidly. For example, gastrointestinal stromal tumours, which have been reported in the horse, are well recognised in human medicine, and in these cases gain-of-function mutations or amplification of c-kit is often observed. Indeed, gastrointestinal stromal tumours are now graded by c-Kit expression and mitotic index to determine the degree of malignancy and whether specific therapy is required. Studies can be carried out that examine the role of various possible candidate genes such as RET, endothelin, and c-kit in gastrointestinal diseases.

Collaborative initiatives between researchers in veterinary and human medicine are to be welcomed because both have much to gain. Work in different species holds promise to elucidate disease causality and pathogenesis, particularly for disorders that have proved difficult to study in humans-namely, motility disorders such as irritable bowel syndrome. Although there are obvious differences between humans and animals in terms of anatomy and diet, the pathophysiological and clinical parallels may be greater than previously thought. Comparative study can encompass investigation of animal intestinal tissues (abattoir derived and clinical material) and surgically resected human tissue. An advantage of this approach is that the use of experimental animals can be minimised. Once normal physiology is better understood, direct pathological comparisons can be made both within and between different species.

\section{Conclusions}

Many benefits exist in the study of both the human and animal forms of disease. We have highlighted some of the common elements in gastrointestinal diseases across 
species and discussed some of the research approaches used in their investigation. Recent initiatives that bear this out are the establishment by the Medical Research Council of the Comparative Clinical Science Panel to foster research links between human and veterinary medicine, and scientific symposia in comparative medicine organised by bodies such as the Royal Society of Medicine. Many clinical benefits are also to be derived from collaboration between the professions in terms of diagnostics and therapeutics (both medical and surgical). In gastroenterology, these have included improved surgical techniques, management of adhesions, advances in critical care, drug therapies, and parenteral nutrition. The direction of this knowledge transfer has traditionally been from human to veterinary medicine, but it is now clear that the movement of information can be two way, with mutual benefit.

We thank W H Tremaine for providing figure 1.

Contributors: NPHH conceived the idea for this clinical review, which was written in part by both authors. NPHH will act as guarantor.

Funding: NPHH is supported by the Dowager Countess Eleanor Peel Trust. The work undertaken by MGD and co-investigators is supported by grants from Cancer Research UK, Medical Research Council, Scottish Executive, Wellcome Trust, Association for International Cancer Research, and the National Association for Colitis and Crohn's Disease.

Competing interests: None declared.

1 Turnage RH Bergen PC. Intestinal obstruction and ileus. In: Feldman $\mathrm{MD}$, Scharschmidt BC, Sleisenger MH, eds. Gastrointestinal and liver disease. Philadelphia: WB Saunders, 1998:1799-810.

2 Kohn CW. Intestinal obstruction. In: Anderson N, Sherding R, Merritt A, Whitlock R, eds. Veterinary gastroenterology. Philadelphia: Lea and Febiger, 1992:173-210.

3 Kamm MA. Why the enteric nervous system is important to clinicians. Gut 2000;47(suppl IV):iv8-9.

4 De Giorgio R, Sarnelli G, Corinaldesi R, Stanghellini V. Advances in our understanding of the pathology of chronic intestinal pseudo-obstruction. Gut 2004;53:1549-52.

5 Mayer EA, Collins SM. Evolving pathophysiologic models of functional gastrointestinal disorders. Gastroenterology 2002;122:2032-48.

Washabau RJ, Stalis IH. Alterations in colonic smooth muscle function in cats with idiopathic megacolon. AmJ Vet Res 1996;57:580-7.

7 Guilford WG. Motility disorders of the bowel. In: Guilford WG, Center SA, Strombeck DR, Williams DA, Mever DJ, eds. Strombeck's small animal gastroenterology. 3rd ed. Philadelphia: WB Saunders, 1996:532-9.
gat
8 Mann SD, Debinski HS, Kamm MA. Clinical characteristics of chronic idiopathic intestinal pseudo-obstruction in adults. Gut 1997;41:675-81.

9 Heaton ND, Garrett JR, Howard ER. The enteric nervous system: structure and pathology. In: Bannister R, ed. Autonomic failure: a textbook of clinical disorders of the autonomic nervous system. 2nd ed. Oxford: Oxford University Press, 1988-238-63.

10 Cottrell DF, McGorum BC, Pearson GT. The neurology and enterology of equine grass sickness: a review of basic mechanisms. Neurogast Motil 1999;11:79-92.

11 De Giorgio R, Guerrini S, Barbara G, Cremon C, Stanghellini V, Corinaldesi R. New insights into human enteric neuropathies. Neurogast Motil 2004;16(suppl 1):143-7.

12 White NA. Epidemiology and etiology of colic In: White NA, ed. The equine acute abdomen. Philadelphia: Lea and Febiger, 1990:49-64.

13 Simpson JW. Diet and large intestinal disease in dogs and cats. J Nutr 1998;128:S2717-22.

14 Bourdu S, Dapoigny M, Chapuy E, Artigue F, Vasson M-P, Dechelotte P, et al. Rectal instillation of butyrate provides a novel clinically relevant model of noninflammatory colonic hypersensitivity in rats. Gastroenterology 2005; $128: 1996-2008$

15 Schwarz NT, Kalff JC, Turler A, Speidel N, Grandis JR, Billiar TR, et al. Selective jejunal manipulation causes postoperative pan-enteric inflammation and dysmotility. Gastroenterology 2004;126:159-69.

16 Little D, Tomlinson JE, Blikslager AT. Post operative neutrophilic inflammation in equine small intestine after manipulation and ischaemia. Equine Vet J 2005:37:329-35.

17 Hunt JM, Edwards GB, Clarke KW. Incidence, diagnosis and treatment of postoperative complications in colic cases. Equine Vet J 1986;18: 264-70.

18 Craven M, Simpson JW, Ridyard AE, Chandler ML. Canine inflammatory bowel disease: retrospective analysis of diagnosis and outcome in 80 cases (1995-2002).J Sm Anim Prac 2004;45:336-42.

19 Satsangi J, Morecroft J, Shah NB, Nimmo E. Genetics of inflammatory bowel disease: scientific and clinical implications. Best Pract Res Clin Gastr 2003;17:3-18.

20 Vanderwinden J, Rumessen JJ. Interstitial cells of Cajal in human gut and gastrointestinal disease. Microsc Res Tech 1999;47:344-60.

21 Hudson NPH, Mayhew IG, Pearson GT. A reduction in interstitial cells of Cajal in horses with equine dysautonomia (grass sickness). Auton Neurosci: Basic Clin 2001·92:37-44.

22 Fintl C, Hudson NPH, Mayhew IG, Edwards GB, Proudman CJ, Pearson GT. The interstitial cells of Cajal (ICC) in equine colic: an immunohistochemical study of horses with obstructive disorders of the small and large intestines. Equine Vet J 2004;36:474-9.

23 Brown DR, Timmermans J-P. Lessons from the porcine enteric nervous system. Neurogast Motil 2004;16(suppl 1):50-4.

24 Kubota M, Ito Y, Ikeda K. Membrane properties and innervation of smooth muscle cells in Hirschsprung's disease. Am J Physiol 1983;244:G406-15

25 Hudson NPH, Mayhew IG, Pearson GT. Presence of in vitro electrical activity in the ileum of horses with enteric nervous system pathology: equine dysautonomia (grass sickness). Auton Neurosci: Basic Clin 2002;99:119-26

(Accepted 6 September 2005)

\section{A memorable patient}

\section{Once there is hope, there is life}

In May 2004 I was part of an international team that responded to an outbreak of Ebola haemorrhagic fever in South Sudan. In this outbreak the astuteness of the local medical staff and the rapid international response resulted in relatively few deaths. Public health doctors like myself were allowed contact with recovered patients, two of whom joined us in our social mobilisation and health education campaigns. One of them was a bright teenager in his final year of secondary school who had lost his mother to the Ebola virus.

He was quickly integrated into the social mobilisation team and came with a powerful message, which he delivered eloquently. He told his story through our loudspeakers carried around the villages, in churches, schools, and markets. Crowds came out to listen to the child who had survived the deadly Ebola virus. His presence reinvigorated the team, strengthened our message, and contributed substantially to controlling the outbreak.

With the outbreak over, we packed up to leave. Our former patient and colleague promised to keep in touch, and he did. Initially I replied to each mail enthusiastically, trying to inspire him to rise above the enormous obstacles in the path to success in South Sudan. However, once I was back to my daily routine in Britain, investigating small outbreaks of gastrointestinal illness, South Sudan quickly became a distant memory. But his mails continued; he sought help to go to university; he wanted to be a doctor, to be like us.

There were no universities in South Sudan, and Khartoum was out of reach for those in the south. The best hope was to move to Uganda, a long trek indeed. Sadly, I could do little more, my replies became less frequent and eventually stopped.

I heard on the news that peace was close at hand between South Sudan (or New Sudan as they call it) and Sudan. I prayed for my teenage friend and for all the children in South Sudan. Decades of war and unrest had robbed them of a chance of an education. I prayed for the people of Darfur, that they too may have peace. I prayed that they might be spared the ravages of Ebola epidemics. I took up contact again with my friend, with a more hopeful tone. Once there is hope, there is life.

Chikwe Ihekweazu fellow of the European Programme for Intervention Epidemiology (EPIET), Health Protection Agency, South West, Stroud (chikwe.ihekweazu@hpa.org.uk) 